\section{Cost-Effectiveness of Mohs Micrographic Surgery versus Surgical Excision for Basal Cell Carcinoma of the Face}

\author{
B.A. Essers, C.D. Dirksen, \\ F.H.M. Nieman, N.W.J. Smeets, \\ G.A.M. Krekels, M.H. Prins, \\ A.M. Neumann \\ University Hospital Maastricht, \\ Maastricht, The Netherlands \\ [Archives of Dermatology 2006;142:187-194]
}

Objective: To assess the cost-effectiveness of Mohs micrographic surgery (MMS) compared with the surgical excision for both primary and recurrent basal cell carcinoma (BCC). Design: A costeffectiveness study performed alongside a prospective randomized clinical trial in which MMS was compared with surgical excision. Setting: The study was carried out from 1999 to 2002 at the dermatology outpatient clinic of the University Hospital Maastricht, Maastricht, the Netherlands. Participants: A total of 408 primary (374 patients) and 204 recurrent (191 patients) cases of facial BCC were included. Main Outcome Measures: The mean total treatment costs of MMS and surgical excision for both primary and recurrent $\mathrm{BCC}$ and the incremental cost-effectiveness ratio, calculated as the difference in costs between MMS and surgical excision divided by their difference in effectiveness. The resulting ratio is defined as the incremental costs of MMS compared with surgical excision to prevent 1 additional recurrence. Results: Compared with surgical excision, the total treatment costs of MMS are significantly higher (cost difference: primary BCC, EUR 254; 95\% confidence interval, EUR 181-324; recurrent BCC, EUR 249; 95\% confidence interval, EUR 175-323). For primary BCC, the incremental cost-effectiveness ratio was EUR 29,231, while the ratio for recurrent $\mathrm{BCC}$ amounted to EUR 8,094. The acceptability curves showed that, for these ratios, the probability of MMS being more cost-effective than surgical excision never reached $50 \%$. Conclusions: At present, it does not seem cost-effective to introduce MMS on a large scale for both primary and recurrent BCC. However, because a 5-year period is normally required to determine definite recurrence rates, it is possible that MMS may become a cost-effective treatment for recurrent BCC.

\section{The Gene for a Rare \\ Autosomal Dominant Form of Pompholyx Maps to \\ Chromosome 18q22.1-18q22.3}

J.-J. Chen, Y.-H. Liang, F.-S. Zhou,

S. Yang, J. Wang, P.-G. Wang, W.-H. Du, S.-J. Xu, W. Huang, X.-J. Zhang

Anhui Medical University, Anhui, China [Journal of Investigative Dermatology 2006;126:300-304]

Pompholyx is a rather common disorder characterized by recurrent crops of vesicles or bullae on the lateral aspects of the fingers, as well as the palms and soles with non-erythematous skin. Until now, very few large families have been reported, so no gene or locus has been identified. Here, we performed a genome-wide search in a large Chinese family to map the chromosome location of the responsible gene. We identified a locus at chromosome 18q22.1-18q22.3 with a maximum two-point LOD score of 3.61 at marker D18S1131 (y1/40.00). Haplotype analyses indicated that the disease gene is located within a 12.07$\mathrm{cM}$ region between markers D18S465 and D18S1362, which corresponds to $8.0 \mathrm{Mb}$. This is the first locus identified for pompholyx. It will aid future identi- fication of the responsible gene, which will be useful for the understanding of the molecular mechanism of pompholyx.

\section{Squamous-Cell Carcinoma in Organ-Transplant Recipients: Results of a Randomized- Controlled Trial}

\begin{abstract}
Y. G.L. de Graaf, C. Kennedy,
R. Wolterbeek, A.F.S. Collen,

R. Willemze, J.N. Bouwes Bavinck

Leiden University Medical Center, Leiden, The Netherlands

[Journal of Investigative Dermatology 2006;126:569-574]
\end{abstract}

A randomized-controlled trial with paired observations was performed with 40 organ transplant recipients to assess the preventive effect of photodynamic therapy (PDT) on the development of new squamous-cell carcinomas and to evaluate the effect of PDT on the number of keratotic skin lesions. The treatment area consisted of a randomly assigned forearm and the corresponding hand, whereas the other forearm and hand served as the control area. After the initial visit, follow-up visits were scheduled at 3-monthly intervals during 2 years. No statistically significant difference was found in the occurrence of new squamous-cell carcinomas between the treated and untreated arms: after 2 years of follow-up, we observed 15 squamouscell carcinomas in 9 out of 40 PDT-treated arms and 10 squamous-cell carcinomas in 9 out of 40 control arms. The number of keratotic skin lesions increased in both arms, but was less pronounced in the PDT-treated arm. After 1 year of follow-up, a trend in favor of the PDT-treated arm was observed, but statistical significance was not reached. Nearly $80 \%$ of the patients reported mild to severe adverse effects consisting of pain and a burning sensation, immediately after the treatment. No long-term adverse events were noted. In conclusion, PDT does not appear to prevent the occurrence of new squamous-cell carcinomas in organ transplant recipients but, to some degree, reduces the increase in keratotic skin lesions. 


\section{Presence of Chimeric Maternally Derived Keratinocytes in Cutaneous Inflammatory Diseases of Children: The Example of Pityriasis Lichenoides}

K. Khosrotehrani, S. Guegan, S. Fraitag, M. Oster, Y. de Prost, C. Bodemer, S. Aractingi

Hôpital Tenon, Paris, France

[Journal of Investigative Dermatology 2006;126:345-348]

During pregnancy, maternal cells may enter the fetal circulation and persist until adulthood. The fate of these cells remains unknown. As unexplained T-cell-mediated conditions such as pityriasis lichenoides (PL) may occur in children, we aimed at identifying maternal cells in lesional skin of PL and controls. Archived skin biopsy specimens from young males with PL, atopic dermatitis or normal skin were scanned for the presence of female (presumably maternal) cells using fluorescence in situ hybridization (FISH) with X- and Y-chromosome-specific probes. Phenotyping of maternal cells relied on FISH combined with anti-CD45, anti-CD1a or anticytokeratin labeling, identifying leukocytes, Langerhans cells and keratinocytes, respectively. Maternal cells were found in PL (11/12) and controls (4/7), but their average frequency was higher in PL: 99 per million cells as compared to 5 per million cells in controls $(\mathrm{p}=$ 0.005). In the epidermis, the maternal microchimeric cells were labeled by anticytokeratin in all cases. We identified maternally derived keratinocytes in the skin of male children with inflammatory skin disorders. These cells may either help repair the damaged skin or home initially in the skin and trigger a host (child) versus graft (mother) disease.

\section{The PINE Study of Epidural \\ Steroids and Local \\ Anaesthetics to Prevent \\ Postherpetic Neuralgia: \\ A Randomised Controlled Trial}

\author{
A.J.M. van Wijck, W. Opstelten, \\ K.G.M. Moons, G.A. van Essen, \\ R.J. Stolker, C.J. Kalkman, \\ T.J.M. Verheij \\ University Medical Center, Utrecht, \\ The Netherlands \\ [Lancet 2006;367:219-224]
}

Background: Postherpetic neuralgia is the most frequent complication of herpes zoster. Treatment of this neuropathic pain syndrome is difficult and often disappointing. We assessed the effectiveness of a single epidural injection of steroids and local anaesthetics for the prevention of postherpetic neuralgia in older patients with herpes zoster. Methods: We randomly assigned 598 patients older than 50 years, with acute herpes zoster (rash $<7$ days) below dermatome C6, to receive either standard therapy (oral antivirals and analgesics) or standard therapy with one additional epidural injection of $80 \mathrm{mg}$ methylprednisolone acetate and $10 \mathrm{mg}$ bupivacaine. The primary end point was the proportion of patients with zoster-associated pain 1 month after inclusion. Analyses were by intention to treat. This study is registered as an International Standard Randomised Controlled Trial, No. ISRCTN32866390. Findings: At 1 month, 137 (48\%) patients in the epidural group reported pain compared with $164(58 \%)$ in the control group (relative risk 0.83 , $95 \%$ CI $0.71-0.97, \mathrm{p}=0.02)$. After 3 months these values were $58(21 \%)$ and $63(24 \%)$, respectively $(0.89,0.65-1.21$, $\mathrm{p}=0.47)$ and, at 6 months, $39(15 \%)$ and $44(17 \% ; 0.85,0.57-1.13, \mathrm{p}=0.43)$. We detected no subgroups in which the relative risk for pain 1 month after inclusion substantially differed from the overall estimate. No patient had major adverse events related to epidural injection. Interpretation: A single epidural injection of steroids and local anaesthetics in the acute phase of herpes zoster has a modest effect in reducing zoster-associated pain for 1 month. This treatment is not effective for the prevention of longterm postherpetic neuralgia.

\section{Interstitial Granulomatous Dermatitis Associated with the Use of Tumor Necrosis Factor Alpha Inhibitors}

A. Deng, V. Harvey, B. Sina, D. Strobel,

A. Badros, J.M. Junkins-Hopkins,

A. Samuels, M. Oghilikhan, A. Gasparini

University of Maryland, Baltimore, Md., USA

[Archives of Dermatology 2006;142:198-202]

Background: Tumor necrosis factor $\alpha(\mathrm{TNF}-\alpha)$ has been implicated in the pathogenesis of numerous inflammatory and autoimmune disorders. Accordingly, TNF- $\alpha$ inhibitors, such as thalidomide, infliximab (Remicade), adalimumab (Humira) and etanercept (Enbrel), have been used with success in the treatment of autoimmune disorders, including psoriasis, rheumatoid arthritis, inflammatory bowel diseases and lymphoproliferative disorders. Although anti-TNF- $\alpha$ therapy is safe and well tolerated, various adverse cutaneous reactions have been reported. Observations: We encountered 5 patients who developed erythematous annular plaques on the trunk and extremities while receiving 4 different medications with inhibitory activity against TNF- $\alpha$. One patient was treated with lenalidomide (Revlimid) for multiple myeloma, 2 received infliximab, and 1 received etanercept for severe rheumatoid arthritis; the last patient was in a clinical trial of adalimumab for psoriatic arthritis. Skin biopsy specimens revealed diffuse interstitial granulomatous infiltrates of lymphocytes, histiocytes and eosinophils, palisading degenerated collagen. Withdrawal of the medications led to complete resolution of the skin lesions. Conclusion: Interstitial granulomatous dermatitis should be considered in the differential diagnosis of skin lesions occurring in the setting of anti-TNF- $\alpha$ therapy. 


\section{Eosinophilic Cellulitis-Like Reaction to Subcutaneous Etanercept Injection}

H. Winfield, E. Lain, T. Horn, J. Hoskyn

University of Arkansas for Medical

Sciences, Little Rock, Ark., USA

[Archives of Dermatology 2006;142:218-220]

Background: Injection site reactions are well recognized in patients treated with etanercept. Previous reports describe histologic findings of a cell-mediated $\mathrm{Th} 1$ reaction, with $\mathrm{CD} 8+\mathrm{T}$ cells composing the majority of the dermal infiltrate. Observations: A pruritic, erythematous, edematous patch occurred on the right thigh of a 57-year-old white woman treated for rheumatoid arthritis within 12-24 h after her second dose of subcutaneous etanercept. The patient had had a similar reaction to adalimum$\mathrm{ab}$ injection 2 weeks prior to presentation. While benzyl alcohol is present in the etanercept preparation, and mannitol in both drugs, dermal injection revealed no reaction to these additives. Biopsy specimens from the etanercept injection site demonstrated papillary dermal edema accompanied by a brisk polymorphous infiltrate with a predominance of eosinophils and scattered flame figures. Conclusions: Histologic features of eosinophilic cellulitis as a response to etanercept have not been reported to date. Although most injection site reactions contain $\mathrm{T}$ cells and represent a Th1 immune response, the findings we report suggest a Th2-mediated phenomenon.

\section{Modulation of Lymphocyte Phenotype and Function by Immunoglobulins}

J. Kirschbaum, K. Forschner, C. Rasche, M. Worm

Charité-Universitätsmedizin Berlin, Berlin, Germany

[British Journal of Dermatology 2006;154:225230]

Background: Immunoglobulins have immune-modulating capacities and are used for the treatment of different dermatological diseases. They have also been reported for the treatment of severe atopic dermatitis (AD). Objectives: To determine the effects of immunoglobulins on the phenotype and function of peripheral $\mathrm{T}$ and $\mathrm{B}$ lymphocytes from patients with $\mathrm{AD}$ in comparison with healthy donors (HD) as controls. Methods: We studied lymphocyte activation and T-cell cytokine production from 12 patients with AD and 10 HD by multicolour flow-cytometric analysis in the presence of immunoglobulins. Results: Immunoglobulins significantly inhibited T-cell activation (CD69), by $71 \%$ (AD) and by $62 \%$ (HD). Production of $\gamma$-interferon and interleukin 4 was also significantly inhibited, by $44 \% / 24 \%$ (AD) and 38\%/10\% (HD), respectively. In addition, $\mathrm{CD} 86$ expression on $\mathrm{B}$ lymphocytes was down-regulated by $30 \%$ in $\mathrm{AD}$ and by $29 \%$ in HD, whereas CD23 expression was decreased without reaching statistical significance. Conclusions: Our data demonstrate that, in vitro, immunoglobulins modulate the activation and cytokine production of peripheral blood lymphocytes from both HD and patients with $\mathrm{AD}$.

\section{Confluent and Reticulate Papillomatosis (Gougerot- Carteaud Syndrome): \\ A Minocycline-Responsive Dermatosis without Evidence for Yeast in Pathogenesis - A Study of 39 Patients and a Proposal of Diagnostic Criteria}

M.D. Davis, R.H. Weenig, M.J. Camilleri

Mayo Clinic, Rochester, Minn., USA

[British Journal of Dermatology 2006;154:287293]

Background: Confluent and reticulate papillomatosis (CRP; GougerotCarteaud syndrome) is a disorder that has been characterized in only small cohorts of patients. Objectives: To better characterize the clinical and pathological findings of the disorder. Methods: We retrospectively reviewed the clinical presentation, response to treatment and histological findings of patients presenting to the Mayo Clinic (Rochester, Minn., USA) with CRP. Results: The disorder was diagnosed in 39 patients between 1972 and 2003. The mean age at onset of the skin eruption was 15 years (range 8-32); 21 patients (54\%) were male; most were white; most (33) pre- sented for reasons of cosmesis, and $8 \mathrm{de}$ scribed the rash as mildly pruritic. At presentation, the skin eruption had been present for a mean of 3.1 years (range 3 months to 20 years) and had been recalcitrant to treatment, including antifungal treatment. Typical objective findings were scaling brown macules and patches and velvety papules and plaques, reticulated and papillomatous at least in part, involving the upper trunk, axillae and neck. The most frequent initial diagnostic impressions were tinea versicolor, acanthosis nigricans and CRP. Scales in 32 cases were examined with potassium hydroxide: 8 (25\%) showed hyphae, and 24 (75\%) did not. Skin biopsy specimens from 21 patients showed variable degrees of hyperkeratosis, acanthosis and papillomatosis. Minocycline was prescribed for 22 patients, of whom 14 of $18(78 \%)$ had complete clearing of the skin eruption and $4(22 \%)$ a partial response. The skin eruptions recurred after stopping treatment in 6 patients. Conclusions: CRP occurs predominantly in young adults and teenagers, with cosmetically displeasing brown scaling patches and plaques affecting the neck, upper trunk and axillae. Frequently, the diagnosis is delayed and the disorder not recognized by physicians, including dermatologists. Clinically, the eruption is most often confused with tinea versicolor. Potassium hydroxide staining of the scale is negative in the majority of cases, implying that fungi are not involved in the pathogenesis of this condition, as has been previously proposed. It is important to recognize this disorder, because minocycline therapy is highly effective in most patients. Criteria for the diagnosis are proposed. 


\section{The Relationship between Neutrophilic Dermatosis of the Dorsal Hands and Sweet Syndrome: Report of 9 cases and Comparison to Atypical Pyoderma Gangrenosum}

H.W. Walling, C.J. Snipes, P. Gerami, W.W. Piette

University of Iowa, Iowa City, Iowa, USA

[Archives of Dermatology 2006;142:57-63]

Background: Neutrophilic dermatoses are a collection of diseases with varying presentation unified by clinical and histologic features. Neutrophilic dermatosis of the dorsal hands is a recently described clinical entity and an evolving disease concept. Its relationship to acute febrile neutrophilic dermatosis (Sweet syndrome), pyoderma gangrenosum and a primary vasculitis has been debated. Observations: We present 9 cases ( 8 women and 1 man) of neutrophilic dermatosis of the dorsal hands, all with consistent histologic features. Two cases had histologic evidence of vasculitis, and 3 had clinical extension of lesions onto the forearms. Most showed fever, leukocytosis and/or an elevated erythrocyte sedimentation rate. Individual cases were associated with leukemia, lung carcinoma and inflammatory bowel disease. All 9 patients responded to systemic corticosteroid therapy, with additional response to dapsone, methotrexate and potassium iodide therapies in several cases. Of the 9 patients, 5 showed complete resolution of their skin disease, whereas 4 required ongoing therapy. We assessed the 43 cases previously reported in the literature. Conclusion: The clinical presentation, laboratory data, histologic features and response to corticosteroid therapy offer strong evidence that neutrophilic dermatosis of the dorsal hands is a localized variant of Sweet syndrome and is also identical to atypical pyoderma gangrenosum when that condition presents on the hands.

\section{Lichen Planus Is Associated with Human Herpesvirus Type 7 Replication and Infiltration of Plasmacytoid Dendritic Cells}

H.J. de Vries, J. van Marle,

M.B. Teunissen, D. Picavet,

F. Zorgdrager, J.D. Bos, J. Weel,

M. Cornelissen

University of Amsterdam, Amsterdam, The Netherlands

[British Journal of Dermatology 2006;154: 361-364]

Background: Lichen planus (LP) is a common inflammatory skin disease of unknown aetiology. Viral causes have been suggested. Objectives: To find candidate viruses associated with LP. Methods: Lesional and non-lesional skin samples, peripheral blood mononuclear cells and serum were obtained from patients with LP. Ultrastructural, viral DNA, immunohistochemical and serological analyses were performed, and comparisons were made with psoriatic and normal skin. Results: Electron microscopy revealed typical 120 - to $200-n m$ enveloped particles with a 100-nm nucleus resembling human herpesvirus (HHV) virions both in the dermis and epidermis of lesional LP tissue. HHV-7 DNA was found in 11 of 18 lesional LP samples, as opposed to only 1 of 11 non-lesional LP samples $(p=0.06), 2$ of 11 lesional psoriasis samples $(p=0.05)$ and none of 4 normal skin samples. No relation was found between LP skin and DNA of other known HHVs (HHV-1 to -6 and -8). With immunohistochemistry, significantly more HHV-7+ cells were found in lesional LP epidermis than in normal epidermis. Lesional LP dermis contained significantly more HHV-7+ cells than non-lesional LP, psoriatic or normal dermis. Moreover, LP skin contained overwhelmingly and consistently more plasmacytoid dendritic cells (upregulated in virally induced conditions) than non-lesional LP samples. Conclusions: We conclude that HHV-7 replicates in LP lesions, but not in psoriasis, another inflammatory skin condition. HHV-7 is possibly involved in the pathogenesis of LP. These preliminary data make further research on this topic of interest.

\section{Cutaneous IgA-Associated Vasculitis Induced by Alcohol}

I.C. Chua, C.R. Aldridge, A.Y. Finlay, P.E. Williams

University Hospital of Wales, Cardiff, UK

[British Journal of Dermatology 2005; 153:1037-1040]

A patient with elevated levels of serum IgA developed purpuric lesions histologically resembling Henoch-Schönlein purpura brought on by consuming alcohol. Alcohol challenge with 5 units of alcohol reproduced the lesions, with a rapid rise of circulating CD4+ and CD8+ $\mathrm{T}$ cells followed by a fall of serum $\mathrm{IgA}$ and $\mathrm{C} 3$ concentration. The skin lesions and serum abnormalities resolved spontaneously within 6 weeks of the alcohol challenge. 